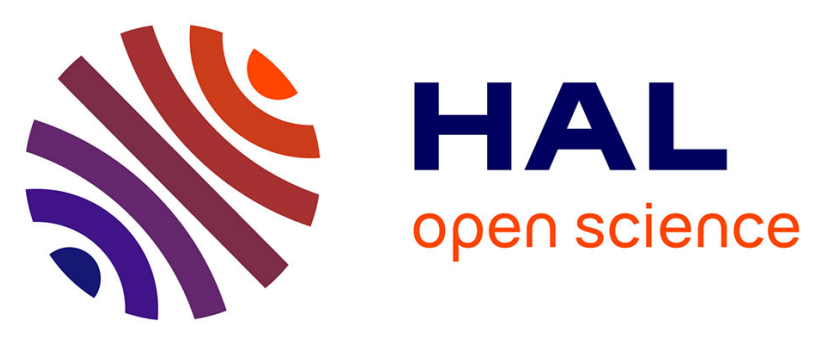

\title{
Le Plateau de Lannemezan
}

Jacques Hubschman

\section{To cite this version:}

Jacques Hubschman. Le Plateau de Lannemezan. Bulletin de l'Association française pour l'étude du quaternaire, 1975, 12 (3-4), pp.207-209. 10.3406/quate.1975.1270 . hal-02735874

\section{HAL Id: hal-02735874 \\ https://hal-univ-tlse2.archives-ouvertes.fr/hal-02735874}

Submitted on 2 Jun 2020

HAL is a multi-disciplinary open access archive for the deposit and dissemination of scientific research documents, whether they are published or not. The documents may come from teaching and research institutions in France or abroad, or from public or private research centers.
L'archive ouverte pluridisciplinaire HAL, est destinée au dépôt et à la diffusion de documents scientifiques de niveau recherche, publiés ou non, émanant des établissements d'enseignement et de recherche français ou étrangers, des laboratoires publics ou privés. 


\section{Le Plateau de Lannemezan}

\section{Jacques Hubschman}

\section{Abstract}

Description of some soils and weathered deposits of the typical piemont formation of Lannemezan. Discussion about the age and modalities of deposit.

\section{Citer ce document / Cite this document :}

Hubschman Jacques. Le Plateau de Lannemezan. In: Bulletin de I'Association française pour l'étude du quaternaire, vol. 12, n³-4, 1975. pp. 207-209;

doi : https://doi.org/10.3406/quate.1975.1270

https://www.persee.fr/doc/quate_0004-5500_1975_num_12_3_1270

Fichier pdf généré le 19/04/2018 


\section{LE PLATEAU \\ DE LANNEMEZAN}

par Jacques HUBSCHMAN

\section{SUMMARY}

Description of some soils and weathered deposits of the typical piemont formation of Lannemezan. Discussion about the age and modalities of deposit.

Le thème "Plateau de Lannemezan" a déjà été traité sous ses différents aspects au cours d'excursions précédentes : on en trouvera les principaux éléments descriptifs et comptes rendus (de $M$. Icole notamment) dans les Livrets-guides des excursions A 6 et C 12 organisées à l'occasion du Congrès INQUA 1969. De même, on pourra consulter avec profit F. Taillefer (1951), H. Alimen (1964), J. Patin (1967), et M. Icole (1969 a et $b, 1973)$. Tout en présentant les arrêts $n^{\circ} 26,27$ et 28 , on se limitera donc jci à un bref rappel des problèmes majeurs que pose la formation de Lannemezan, en relation avec la mise en place et l'évolution des dépôts quaternaires postérieurs.

\section{1 - Le premier problème, fondamental, concerne l'âge de cette formation}

Rappelons que le Plateau de Lannemezan (figure 21), ancien cône de déjection de la Neste, représente le modèle le plus vaste et le mieux conservé des épandages du piémont nord-pyrénéen: plateaux de Cieutat-Orignac et de Ger, à l'ouest; éléments de plateau entre Garonne et Ariège, à l'est. Postérieure au Pontien qu'elle ravine localement (M. Richard, 1946), la formation, souvent épaisse de plusieurs dizaines de mètres, est composée essentiellement de galets et parfois de blocs emballés dans une matrice plus ou moins

Fig. 21 - Le plateau de Lannemezan dans l'ensemble régional. 1. Pyrćnćcs. 2. Massif Central, bordure sédimentaire et molasses de l'Agenais. 3. Terrains molassiques du Terrefort et de la Gascogne gersoise. 4. Plateau de Lannemezan. 5. Dépression périphérique de la Montagne Noire. 6. Grands couloirs alluviaux.

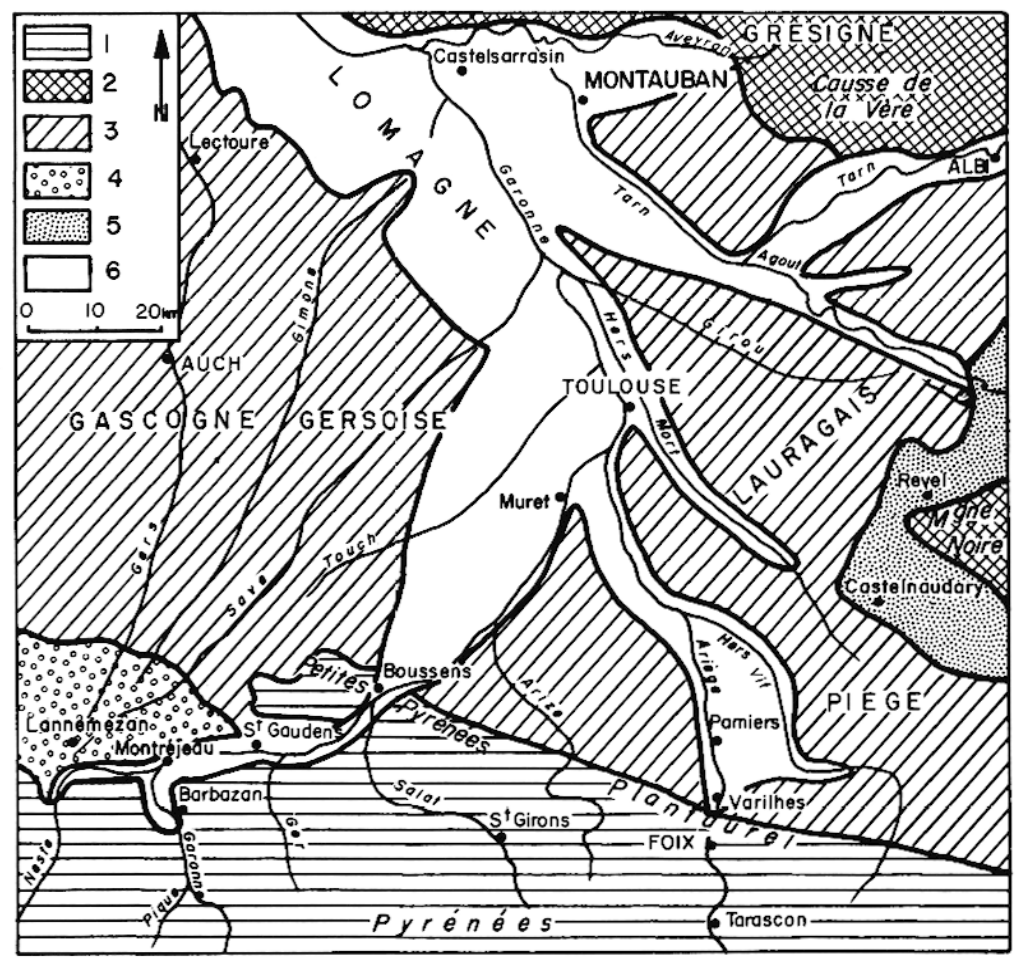



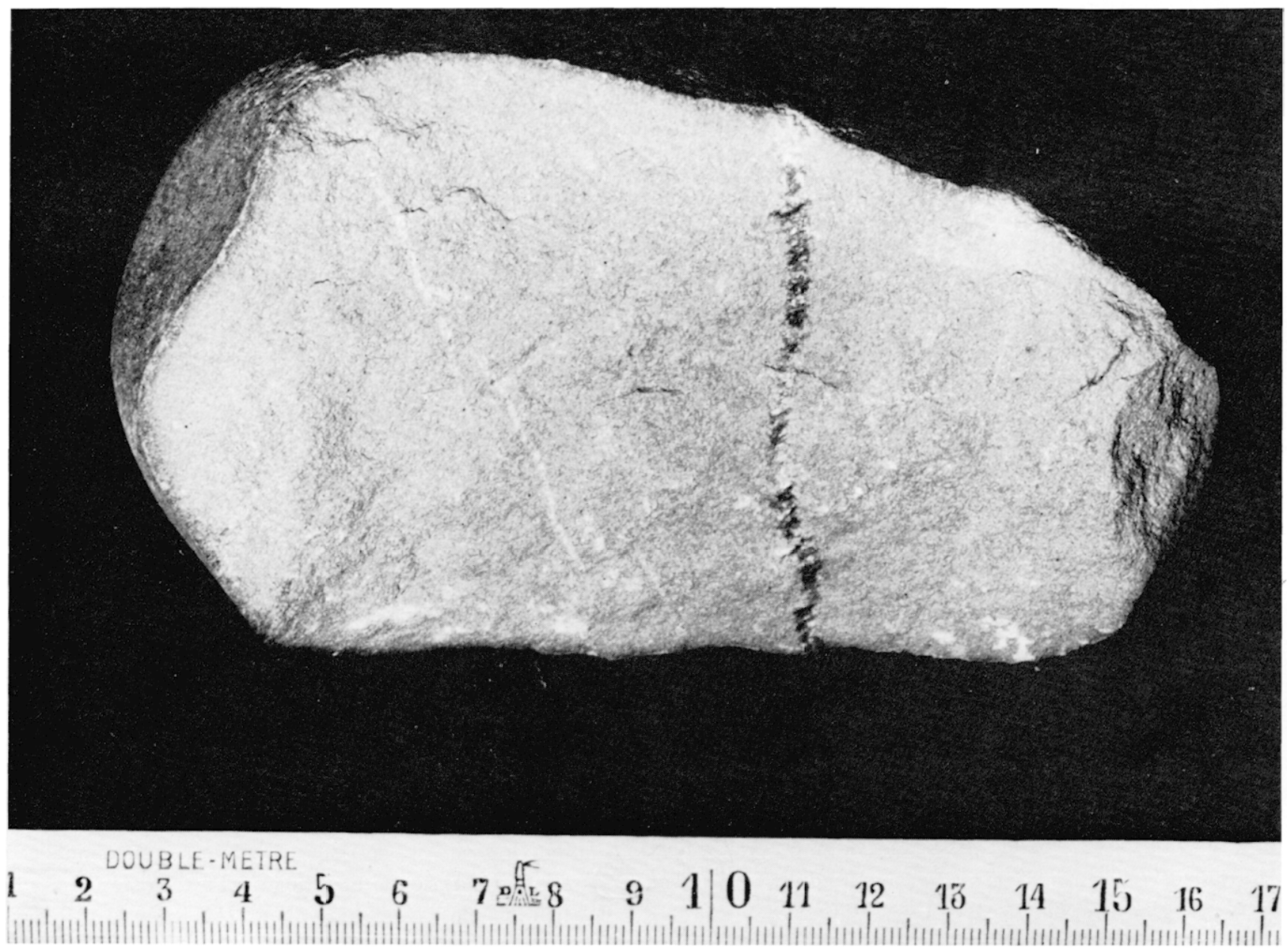

Photo 23 - Le "fossile" du Lannemezan : quartzite totalement grésifié, à patine corticale rouille foncé.

argtleuse et bariolée. L'acidité du milieu, qui a provoqué la disparition des fossiles, la grande variabilité des faciès du Pontien comme de la formation de Lannemezan et l'absence de claires limites stratigraphiques et sédimentologiques entre eux, tout ceci explique que l'âge de ces dépôts ne soit pas connu avec précision. Depuis M. Boule (1895), l'ensemble de ces matériaux sont traditionnellement imputés au Pliocène.

Les travaux de $\mathrm{H}$. Alimen et $\mathrm{M}$. Icole ont cependant montré que la formation de Lannemezan est en réalité complexe et qu'il convient d'y distinguer 2 générations de dépôts :

- d'une part, une formation ancienne, ponticopliocène ou pliocène, véritable soubassement du plateau et qui n'affleure qu'au sommet des larges croupes dominant le paysage. D'allure plutôt hétérométrique quoique dépourvu de très gros blocs, ce matériel présente un spectre pétrographique particulier, portant sur les éléments ayant échappé à l'altération complète : les quartz dominent sur les quartzites et ces derniers sont souvent entièrement grésifiés (grès de Lannemezan) lorsque leur dimention n'est pas excessive (photo 23);
- d'autre part, un dépôt plus récent mais beaucoup plus étendu, d'épaisseur assez constante, qui ravine le Pliocène sous forme de chenaux de mieux en mieux inscrits, vers l'aval, sous la surface du plateau. C'est le Donau, première nappe quaternaire dont le matériau plus grossier que le précédent, se serait déposé avec de très gros blocs (dimension supérieure au $\mathrm{m}^{3}$ ). Dans cette alluvion, les quartzites sont aujourd'hui plus abondants que les quartz et leur cœur reste sain sous un cortex ferrugineux de 1 à $2 \mathrm{~cm}$ d'épaisseur, comme dans le Donau garonnais.

2 - Le mode de mise en place de ces remblaiements découle, pour une part, de l'interprétation chronologique. Pour F. Taillefer (1951) suivi par F. Crouzel (1957), la forme même du Lannemezan "cône torrentiel court et trapu", les caractères sédimentologiques (hétérométrie dominante) et la présence de blocs très volumineux, évoquent plutôt des épandages torrentiels de type mudflow, fonctionnant sous climats franchement semi-arides (1). Pour H. Alimen et M. Icole, en

(1) Au cours de l'excursion 1975, P. Barrère a présenté certains de ces blocs sur le pédoncule qui relie le plateau à la montagne (entre les arrêts $n^{\circ} 25$ et 26 ). 
revanche, le Pliocène de Lannemezan ne fait qu'achever la sédimentation pontienne tandis que le Donau, avec ses gros blocs abandonnés au pied de la montagne, pourrait traduire une mise en place glaciaire.

3 - Le mode d'altération et de pédogénèse s'apparente plus ou moins à celui du Donau garonnais. A proximité de Capvern-les-Bains (maison d'enfants), la coupe de l'arrêt $\mathrm{n}^{\circ} 26$ (1) montre, à 30 ou $40 \mathrm{~m}$ sous la surface du plateau, un faciès de profondeur classique du Lannemezan : argile caillouteuse ocre à quartz et quartzites ferruginisés, à fantômes de galets gneissogranitiques, riche en minéraux kaoliniques bien organisés (pas de gibbsite dans les fantômes de galets cristallins).

A la sortie est de Lannemezan, l'arrêt $n^{\circ} 27$ permet d'observer, à la faveur d'une tranchée routière, le sommet du Donau altéré. Un sol brun-acide humifère (intergrade aux sols de touya) s'est différencié dans un matériel argilo-sableux ocre rougeâtre à quartzites partiellement grésifiés et à fantômes gneisso-granitiques totalement argilisés et intégrés à la matrice. L'aspect et les caractéristiques de cette coupe sont très typiques des zones relativement bien drainées de ce secteur du Plateau de Lannemezan. On notera la faible différenciation du profil superficiel, liée sans doute à la fois au rajeunissement $\mathrm{du}$ profil (proximité de bordure de vallon) et à l'abondance d'Al qui maintient, en milieu acide, le complexe argilo-humique à l'état floculé : de ce point de vue, le contraste est éclatant avec les profils développés dans les couvertures limoneuses géochimiquement mûres.

(1) Coupe non visitée au cours de l'excursion 1975, en raison des mauvaises conditions météorologiques et des impératifs horaires.
Dès le nord de la ville de Lannemezan, le plateau, défoncé par les vallées dissymétriques de l'éventail de rivières gasconnes, n'est plus conservé vers l'aval que sous forme de longues lanières. La carrière de la tuilerie de Blajan (arrêt $n^{\circ} 28$ ) entaille précisément l'un de ces interfluves séparant la vallée de la Gesse de celle de la Bernesse. Ravinant un dépôt argileux marmorisé vraisemblablement pontien, exploité par la carrière, l'alluvion du Donau se présente comme un cailloutis à galets surtout quartzeux, scellé par une couverture limoneuse, l'ensemble offrant 3 à $4 \mathrm{~m}$ d'épaisseur. La différenciation du profil et ses principaux caractères sont très proches de ceux qui marquent le Donau garonnais: sol lessivé hydromorphe à tendance glossique dans les limons; matrice argilo-sableuse ocre emballant les cailloutis, riche en minéraux kaoliniques bien cristallisés et à forte dégradation hydromorphe. Toutefois, la rareté des quartzites, la couleur de fond de la matrice, qui évoque plutôt la teinte caractéristique des altérites du Lannemezan, la relative abondance des minéraux kaoliniques dans la couverture limoneuse, suggèrent que ce Donau, déposé par la Gesse ou la Bernesse, remanie largement le matériel du Plateau de Lannemezan sensu stricto.

4 - En définitive, l'un des enseignements stratigraphiques majeurs de l'étude du Lannemezan, et que l'on peut considérer aujourd'hui comme acquis, concerne le Donau : ce dépôt, sous la forme d'éléments de plateau en bordure de la chaîne ou de nappe alluviale culminante à l'aval, représente le premier remblaiement quaternaire de ce piémont nord-pyrénéen et traduit probablement une certaine rupture dans les conditions bioclimatiques : ainsi peut être grossièrement définie la limite Tertiaire-Quaternaire dans cette région.

\section{Bibliographie}

(Voir p. 214). 